\title{
Quantification of short- and medium-chain chlorinated paraffins in environmental samples by gas chromatography quadrupole time-of-flight mass spectrometry
}

\author{
Wei Gao ${ }^{a}$, Jing $\mathrm{Wu}^{\mathrm{a}}$, Yawei Wang ${ }^{\mathrm{a}, \mathrm{b}, \mathrm{c}, *}$, Guibin Jiang ${ }^{\mathrm{a}}$ \\ a State Key Laboratory of Environmental Chemistry and Ecotoxicology, Research Center for Eco-Environmental Sciences, Chinese Academy of Sciences, \\ Beijing 100085, China \\ ${ }^{\mathrm{b}}$ Institute of Environment and Health, Jianghan University, Wuhan 430056, China \\ ${ }^{\mathrm{c}}$ University of Chinese Academy of Sciences, Beijing 100049, China
}

\section{A R T I C L E I N F O}

\section{Article history:}

Received 26 April 2016

Accepted 29 April 2016

Available online 30 April 2016

\section{Keywords:}

GC-qTOF-HRMS

Chlorinated paraffin

High resolution

\begin{abstract}
A B S T R A C T
Chlorinated paraffins (CPs) are technical products produced and used in bulk for a number of purposes However, the analysis of CPs is challenging, as they are complex mixtures of compounds and isomers. We herein report the development of an analytical method for the analysis of short-chain CPs (SCCPs) and medium-chain CPs (MCCPs) using quadrupole time-of-flight high-resolution mass spectrometry (GCNCI-qTOF-HRMS). This method employs gas chromatography with a chemical ionization source working in negative mode. The linear relationship between chlorination and the $\mathrm{CP}$ total response factors was applied to quantify the $\mathrm{CP}$ content and the congener group distribution patterns. In a single injection, 24 SCCP formula groups and 24 MCCP formula groups were quantified. Extraction of accurate masses using qTOF-HRMS allowed the SCCPs and MCCPs to be distinguished, with interference from other chemicals (e.g., PCBs) being largely avoided. The SCCP and MCCP detection limits were $24-81 \mathrm{ng} / \mathrm{mL}$ and $27-170 \mathrm{ng} / \mathrm{mL}$, respectively. Comparison of the obtained results with analytical results from gas chromatography coupled with electron capture negative ionization low-resolution mass spectrometry (GC-ECNI-LRMS) indicate that the developed technique is a more accurate and convenient method for the analysis of CPs in samples from a range of matrices.
\end{abstract}

(C) 2016 Elsevier B.V. All rights reserved.

\section{Introduction}

Chlorinated paraffins (CPs), also known as polychlorinated $n$ alkanes, are semi-volatile chemicals (SVOCs) [1,2], which have been used in large amounts in various commercial products for several decades $[1,3]$. The commercial CP mixtures can be divided into three categories, namely short-chain chlorinated paraffins (SCCPs) $\mathrm{C}_{10}-\mathrm{C}_{13}$, medium-chain chlorinated paraffins (MCCPs) $\mathrm{C}_{14}-\mathrm{C}_{17}$, and long-chain chlorinated paraffins (LCCPs) C $>17$. SCCPs in particular have drawn significant attention due to their high toxicity [2]; however, as MCCPs and SCCPs coexist in the environment, and MCCPs can be transformed into SCCPs via environmental processes such as combustion, the issue of MCCP analysis should also be addressed.

\footnotetext{
* Corresponding author at: State Key Laboratory of Environmental Chemistry and Ecotoxicology, Research Center for Eco-Environmental Science, Chinese Academy of Sciences, P.O. Box 2871, Beijing 100085, China.

E-mail address: ywwang@rcees.ac.cn (Y. Wang).
}

The quantification of CPs in environmental samples is challenging [4] due to the high complexity of the industrial mixtures and self-interference among the CPs. A number of different methods have been developed to date for the determination of SCCPs and MCCPs in a range of environmental matrices. Tomy et al. applied electron capture negative ionization high-resolution mass spectrometry (ECNI-HRMS) for the analysis of MCCPs and SCCPs $[5,6]$. This technique is well-suited to routine analyses, and has been employed in our laboratory [7]. Recently, two-dimensional gas-chromatography $(\mathrm{GC} \times \mathrm{GC})$ methods have also been applied to separate SCCPs $[8,9]$, as have mathematical methods such as principal component analyses (PCA) and multiple linear regression [10-12]. Alternative methods include the determination of SCCPs by short-column GC/ECNI-MS [13], carbon skeleton reaction gas chromatography [14], and on-line dechlorination-hydrogenation of chlorinated paraffin mixtures using GC and GC/MS [15,16]. However, these methods present several challenges such as high cost and the risk of interference between other chlorinated pollutants and CPs with the same nominal mass. Recently, Bogdal 
et al. developed a novel technique based on the chlorine-enhanced atmospheric pressure chemical ionization (APCI) method, using a quadrupole time-of-flight high-resolution mass spectrometry (APCI-qTOF-HRMS) system for fast quantification of CPs in environmental samples [17]. However, this direct injection method is problematic due to the requirement for rigorous clean-up procedures.

Interference related to mass overlap between SCCP and MCCP congeners must also be addressed, and fragmentation patterns should be studied to allow more accurate quantification of CPs. With these challenges in mind, we herein report the development of a novel analytical approach based on the GC-NCI-qTOF-HRMS system to simultaneously analyze SCCPs and MCCPs in a single injection. High-resolution GC-qTOF was employed to directly quantify SCCPs and to avoid possible interference by MCCPs in environmental samples. Twenty-four different SCCP formula groups $\left(\mathrm{C}_{10}-\mathrm{C}_{13}\right.$ with 5-10 chlorine atoms) and $24 \mathrm{MCCP}$ formula groups $\left(\mathrm{C}_{14}-\mathrm{C}_{17}\right.$ with $5-10$ chlorine atoms) were analyzed by extracting accurate masses. CPs bearing fewer chlorine atoms and shorter chain lengths were also studied. Finally, samples from a range of environmental matrices were analyzed using the developed method.

\section{Materials and methods}

\subsection{Standards and reagents}

Pesticide analytical grade solvents were purchased from J.T. Baker (Phillipsburg, NJ, USA). Solutions of the SCCP mixtures (100 ng/ $\mu \mathrm{L}, \mathrm{C}_{10}-\mathrm{C}_{13}$ with $51 \%, 55.5 \%$, and $63 \%$ chlorination, $100 \%$ purity) and MCCP mixtures ( $100 \mathrm{ng} / \mu \mathrm{L}, \mathrm{C}_{14}-\mathrm{C}_{17}$ with $42 \%, 52 \%$, and $57 \%$ chlorination, $100 \%$ purity) in cyclohexane and $\varepsilon$-hexachlorocyclohexane $(\varepsilon-\mathrm{HCH}$, solution in cyclohexane, $10 \mathrm{ng} / \mu \mathrm{L}, 99.9 \%$ purity) were purchased from Dr. Ehrenstorfer GmbH (Augsburg, Germany). 1,5,5,6,6,10-Hexachlorodecane $\left({ }^{13} \mathrm{C}_{10^{-}}, 100 \mathrm{ng} / \mu \mathrm{L}\right.$ solution in cyclohexane, $\geq 98 \%$ purity $)$ and $1,5,5,6,6,10$-hexachlorodecane (unlabeled, $100 \mathrm{ng} / \mu \mathrm{L}$ in cyclohexane, $\geq 98 \%$ purity) were purchased from Cambridge Isotope Laboratories (Andover, USA). 2,5,6,9-Tetrachlorodecane (10 ng/ $\mu \mathrm{L}$ in cyclohexane, 98.3\% purity) and 1,2,9,10-tetrachlorodecane $(10 \mathrm{ng} / \mu \mathrm{L}$ in cyclohexane, $99.3 \%$ purity) were obtained from Dr. Ehrenstorfer GmbH (Augsburg, Germany). Solutions of $1,1,1,3,9,11,11,11$-octachloroundecane $(100 \mathrm{ng} / \mu \mathrm{L}$ in cyclohexane, $>98 \%$ purity) and $1,1,1,3,10,12,12,12$-octachlorododecane ( $100 \mathrm{ng} / \mu \mathrm{L}$ in cyclohexane, $97.7 \%$ purity) were obtained from Chiron AS, Stiklestadveien, Norway.

\subsection{Sample preparation}

To test the performance of the NCI-qTOF-HRMS method, samples from several environmental matrices were analyzed for both SCCPs and MCCPs. The detailed information of these samples is provided in Supplementary material section Sample Collection and Preparation.

Air samples were obtained using a passive air sampler (XpressApplication Developer, XAD). Organic film samples were collected from dichloromethane-wetted Kimwipes ${ }^{\circledR}$ after wiping a window surface. Food samples were purchased from several well-known fast food outlets. The industrial $\mathrm{CP}$ products were kindly provided by manufacturers. Sample pretreatment was based on our previously reported method $[18,19]$ with some minor modifications. Briefly, frozen dried samples $(1 \mathrm{~g})$ were mixed with diatomaceous earth (5g) and spiked with the ${ }^{13} \mathrm{C}_{10}-1,5,5,6,6,10-$ hexachlorodecane surrogate standard (10 ng) prior to accelerated solvent extraction (ASE). The extract was concentrated to approx- imately $1 \mathrm{~mL}$ by rotary evaporation. The extract was then cleaned and fractionated on a $1.5 \mathrm{~cm}$ silica-Florisil ${ }^{\circledR}$ composite column packed with Florisil ${ }^{\circledR}(3 \mathrm{~g})$, neutral silica gel $(2 \mathrm{~g})$, acidic silica gel ( $5 \mathrm{~g}, 30 \%$ ), and anhydrous sodium sulfate $(4 \mathrm{~g})$ (packed from bottom to top). The column was conditioned with $n$-hexane $(50 \mathrm{~mL})$, and the sample was eluted with $n$-hexane $(40 \mathrm{~mL})$ (fraction 1 contained polychlorinated biphenyls and toxaphenes), followed by dichloromethane $(50 \mathrm{~mL})$ and $n$-hexane $(50 \mathrm{~mL})$ (fraction 2 contained $\mathrm{CPs}$ and $\mathrm{HCHs}$ ). The second fraction was concentrated to approximately $2 \mathrm{~mL}$ by rotary evaporation and further concentrated to close to dryness under a gentle stream of $\mathrm{N}_{2}$. The fraction was then reconstituted in cyclohexane $(200 \mu \mathrm{L})$. Prior to MS analysis, a $\varepsilon-\mathrm{HCH}(10 \mathrm{ng})$ was added as recovery standard to determine the sample recoveries. Instrumental blanks were composed of pure cyclohexane. No CPs were observed following injection of the blanks.

\subsection{Instrumentation}

An Agilent 7200 GC-QTOF mass spectrometer (Agilent Technologies, Santa Clara, USA) operated in negative chemical ionization $(\mathrm{NCI})$ mode and controlled by MassHunter Acquisition B.07 was used in this study. The GC system was equipped with a DB-5MS Ultra Inert $30 \mathrm{~m}(0.25 \mathrm{~mm}$ i.d., $0.25 \mu \mathrm{m}$ thick $)$ capillary column. The GC oven temperature was programmed to begin at $100^{\circ} \mathrm{C}$ (held for $1 \mathrm{~min}$ ), followed by an increase to $160^{\circ} \mathrm{C}$ at a rate of $5^{\circ} \mathrm{C} / \mathrm{min}$ (held for $2 \mathrm{~min}$ ), then an increase to $310^{\circ} \mathrm{C}$ at $30^{\circ} \mathrm{C} / \mathrm{min}$ (held for $12 \mathrm{~min}$ ). Splitless injections $(2 \mu \mathrm{L}$ ) were carried out at $280^{\circ} \mathrm{C}$ using ultrapure helium as the carrier gas at a flow rate of $1.0 \mathrm{~mL} / \mathrm{min}$. The auxiliary heating and ion source temperatures were set at $280^{\circ} \mathrm{C}$ and $150^{\circ} \mathrm{C}$, respectively. A solvent delay of 5 min was used to prevent damage in the ion source filament. QTOF MS was operated at 5 spectra/s in the $m / z$ range $50-600$, with a resolution of approximately 15,000 at $\mathrm{m} / \mathrm{z} 300-600$. Mass spectrometry grade PFTBA (perfluorotri- $n$-butylamine) was used for the daily mass calibration. MassHunter Quantitative Analysis B.07 and MassHunter Qualitative Analysis B.07 were applied for data treatment. The workflow for the chlorination response factorbased quantification method is described in Fig. S1.

When analyzing CPs, it is necessary to distinguish between different congener groups, as they generate a series of ions in the $m / z$ range 300-600. In our previously reported GC-ECNI-LRMS method $[18,19]$, to minimize interference from other CPs (e.g., SCCPs or MCCPs), we carefully determined the retention time range for each congener group and calculated the quantitative and qualitative ion ratios based on the chromatographic and mass spectrometric results. The integration range of the quantitative ion was set by comparison of the peak cluster shape between the quantitative ion and the qualitative ion, which should be within the range of the corresponding standard retention times. We also consulted the work of Reth and Oehme [7] to calculate the quantitative and qualitative ion ratios of each congener in the SCCP and MCCP standards and in the samples employed herein. The congener ratios between the standards and the real samples were compared. Where the obtained ratio fell into the range of the MCCP standard, it was concluded that SCCP congener quantification suffered from interference from the MCCPs. Thus, the integration range end point should be modified according to the starting retention time of the corresponding MCCP congener. Indeed, the quantification method developed herein further reduced interference from the MCCPs [14]. For example, the $\mathrm{C}_{11} \mathrm{H}_{16} \mathrm{Cl}_{8}$ formula group in the SCCP standards gave a quantification ion to qualification ion response ratio of 1.20 , while in the MCCP standards, the $\mathrm{C}_{16} \mathrm{H}_{28} \mathrm{Cl}_{6}$ formula group response ratio was 1.00 . A ratio of $\geq 1.20$ indicated that the total response was generated by the $\mathrm{C}_{11} \mathrm{H}_{16} \mathrm{Cl}_{8}$ formula group; while a ratio of $\leq 1.00$ indicated 

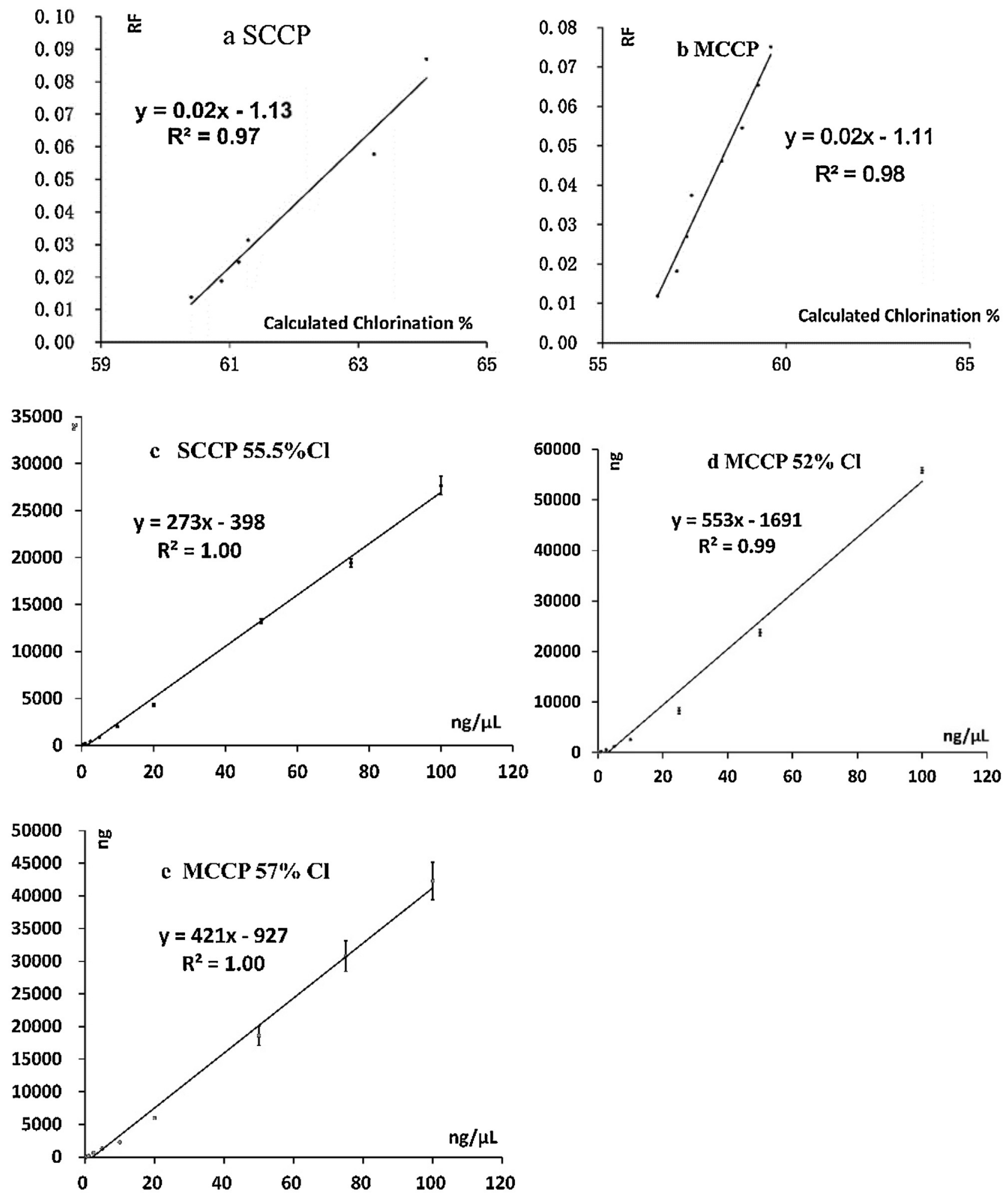

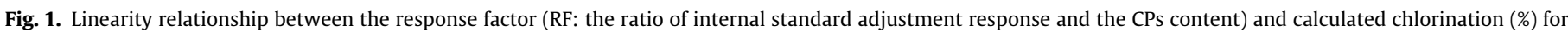
MCCPs and SCCPs; the linearity range of SCCP and MCCP (a) Standard curve of SCCPs at $10 \mathrm{ng} / \mu \mathrm{L}$ (different chlorination obtained by mixing $51.5 \% \mathrm{Cl}, 55.5 \% \mathrm{Cl}$ and $63 \% \mathrm{Cl}$ SCCP standards); (b) standard curve of MCCPs at $10 \mathrm{ng} / \mu \mathrm{L}$ (different chlorination obtained by mixing $42 \% \mathrm{Cl}, 52 \% \mathrm{Cl}$ and $57 \% \mathrm{Cl}$ MCCP standards) Linearity range of SCCPs and MCCPs. (c) Linearity of $55 \%$ chlorinated SCCP mixtures $(0.25-100 \mathrm{ng} / \mu \mathrm{L})$. (d) Linearity of $52 \%$ chlorinated MCCP mixtures $(0.25-100 \mathrm{ng} / \mu \mathrm{L})$. (e) Linearity of $57 \%$ chlorinated MCCP mixtures $(0.25-100 \mathrm{ng} / \mu \mathrm{L})$.

that the $\mathrm{C}_{16} \mathrm{H}_{28} \mathrm{Cl}_{6}$ formula group contributed $100 \%$ towards the response.

For the GC-NCI-qTOF-HRMS method, the SCCP and MCCP contents and congener distributions were derived based on the high-resolution mass spectra recorded in full-scan mode.

\subsection{Auto-integration procedure}

In the GC-ECNI-LRMS method, manual integration is traditionally applied to compare the peak shapes and retention times with 

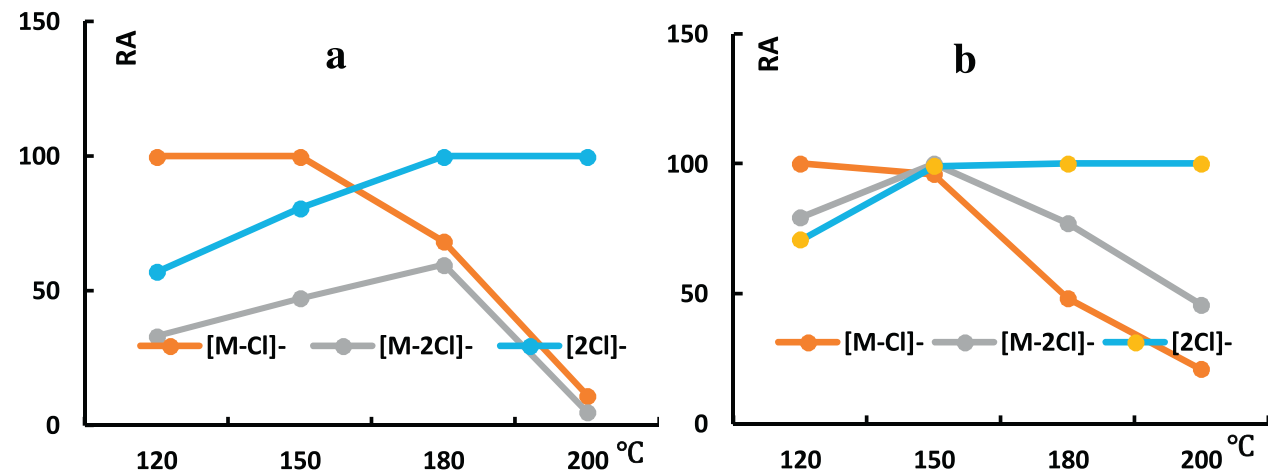

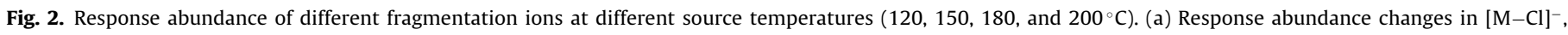

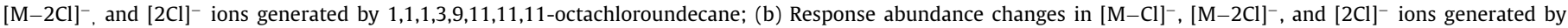
$1,1,1,3,10,12,12,12$-octachlorododecane.

the reference standards. In the NCI-TOF-MS method, the observed EIC (extracted ion chromatography) peak was comparable to that of the standard, as high-resolution MS removed interference from the matrix. Autointegration was applied using MassHunter Quantitative Analysis B.07. The accurate masses of the 96 quantitative and qualitative ions along with their retention times (Tables S1 and S2) were added to the method, and the mass accuracy window was set at $50 \mathrm{ppm}$. In addition, a Universal Integrator was employed. New batch files were built, and the data files were imported. Finally, the integration results were obtained in CSV form.

\section{Results and discussion}

\subsection{Limit of detection and linearity range}

To determine the instrumental limit of detection (LOD) of the GC-NCI-qTOF-HRMS system, five replicate analyses of the CP mixture standards were performed. The LOD was derived from the standard deviation of the signal intensity of the five replicate injections multiplied by Student's T-value at a 95\% confidence level. In practical samples, detection of a congener group was defined as both $m / z$ values of the quantitative and qualitative ions being detected above their respective LODs, and where the LOD of the congener group was equal to the LOD of the least sensitive of the two monitored $m / z$ values. The LOD for the SCCPs and MCCPs was defined as detection of the most abundant congener group. The LOD of the MCCPs was in the range of $27-170 \mathrm{ng} / \mathrm{mL}$, while that of the SCCPs was in the range of $24-81 \mathrm{ng} / \mathrm{mL}$. The LOD of each formula group is provided in Tables S1 and S2. The LOD of the TOF scan was significantly higher than the full scan LRMS results and was comparable to the results obtained under the single ion monitoring (SIM) with the electron multiplier (EM) providing a gain factor of 50. Thus, the LOD obtained under NCI-TOF-MS (SCCP: $24-81 \mathrm{ng} / \mathrm{mL}$, and MCCP: $27-170 \mathrm{ng} / \mathrm{mL}$ ) was comparable to that of the previously reported GC/ECNI-LRMS method [12], and lower than that of the HPLC-APCI-HRMS method [17].

To test the linearity, ten-point curves $(0.25,0.5,1.0,2.5,5.0$, $10,20,50,75$, and $100 \mathrm{ng} / \mu \mathrm{L}$ ) were fitted using standard solutions of $55.5 \% \mathrm{Cl}$ SCCP, $52 \% \mathrm{Cl}$ MCCP, and 57\% Cl MCCP mixtures. Fig. 1 shows the linear ranges of the NCI-qTOF-HRMS response based on weighted linear regression. The linearity range for both SCCP and MCCP was three orders of magnitude, which was higher than that of the GC/ECNI-LRMS method [12]. Considering that TOF-MS usually acts as a screening tool and the linearity range is relatively narrow, these results were satisfactory. This relatively good linearity performance for the CPs was due to no single isomer reaching its upper limit, even at high total concentrations.

\subsection{Accuracy and repeatability}

Accuracy was calculated as the ratio between the average measured concentration $(n=5)$ and the reference SCCP and MCCP mixture standards at different chlorination degrees ( $51.5 \% \mathrm{Cl} \mathrm{SCCP}$, $55.5 \% \mathrm{Cl} \mathrm{SCCP}, 63 \% \mathrm{Cl}$ SCCP, 52\% $\mathrm{Cl} \mathrm{MCCP}$, and 57\% $\mathrm{Cl} \mathrm{MCCP}$ ), as outlined in Eq. (1):

$\operatorname{Accuracy}(\%)=\frac{C_{\mathrm{d}} \times 100}{C_{\mathrm{r}}} \%$

where $C_{d}$ is the average measured concentration of the reference standard and $C_{r}$ is the actual concentration of the reference standard.

As shown in Table 1 relative accuracies of $86-124 \%$ and $114-129 \%$ were acquired by NCI-qTOF-MS for SCCPs and MCCPs, respectively. When using the binary mixture standards of SCCP and MCCP, larger positive bias were acquired than that for the single mixture standard.

Repeatability can be described by the standard deviation of repeated injections ( $n=18$, spiked at 1,10 , and $100 \mathrm{ng} / \mathrm{L}$ of both $55 \%$ $\mathrm{Cl} \mathrm{SCCP}$ and $52 \% \mathrm{Cl}$ MCCP) over a single day (intra-day) and across several days (inter-day). The relative standard deviations (RSDs) of SCCP for the inter-day injections obtained by NCI-TOF-MS at the three concentration levels were $2.55 \%, 1.95 \%$, and $3.58 \%$, respectively. For the MCCP, the corresponding RSDs were $12.3 \%, 7.37 \%$, and $0.97 \%$, respectively.

\subsection{Ion source temperature and fragmentation patterns}

As previously reported, source temperature may influence the relative ion abundances in a system [5,20,21], with higher temperatures increasing the abundance of fragment ions, and lower temperatures boosting molecular ion abundance. According to the work of Tomy et al. [5], the characteristic [M-Cl]- ion reached maximum abundance compared to the non-characteristic $\mathrm{Cl}_{2}-$ and $\mathrm{HCl}_{2}$ - ions at an ion source temperature of $120^{\circ} \mathrm{C}$, and so this temperature was selected to maximize selectivity. As reproducibility of the negative ion mass spectrum is poor [20] between different instruments, we evaluated the possible influence of ion source temperature on response. In this study, CPs were detected at $120^{\circ} \mathrm{C}$, $150^{\circ} \mathrm{C}, 180^{\circ} \mathrm{C}$, and $200^{\circ} \mathrm{C}$ to evaluate the effects of source temperature on the response. As shown in Figs. 2 and S5, the [M-Cl]abundance decreased with increasing ion source temperature, and for the $\mathrm{C}_{12} \mathrm{Cl}_{8}$ single isomer at $180^{\circ} \mathrm{C}$, the $[\mathrm{M}-2 \mathrm{Cl}]-$ abundance exceeded that of the $[\mathrm{M}-\mathrm{Cl}]-$ ion. As shown in Fig. 2, the abundance of the $[\mathrm{M}-\mathrm{Cl}]^{-}$ion was maximized at $120^{\circ} \mathrm{C}$, with very little difference being observed between $120^{\circ} \mathrm{C}$ and $150^{\circ} \mathrm{C}$. For the $\mathrm{C}_{11} \mathrm{Cl}_{8}$ isomer, $[\mathrm{M}-\mathrm{Cl}]^{-}$was still the most abundant ion, while for the 
Table 1

Test performance of the repeatability of the NCI-TOF-HRMS method.

\begin{tabular}{|c|c|c|c|c|c|c|c|}
\hline Performance test & 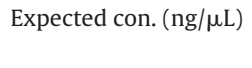 & $\begin{array}{l}\text { Calculated con. } \\
\text { ( } \pm \text { error) } \mathrm{ng} / \mu \mathrm{L}\end{array}$ & Accuracy & Binary mix & $\begin{array}{l}\text { Expected con. } \\
(\mathrm{ng} / \mu \mathrm{L})\end{array}$ & $\begin{array}{l}\text { Calculated con. } \\
\text { ( } \pm \text { error })(n g / \mu L)\end{array}$ & Accuracy ${ }^{a}$ \\
\hline $51 \% \mathrm{Cl} \mathrm{SCCP}$ & $\begin{array}{l}5 \\
.00\end{array}$ & $4.30( \pm 0.41)$ & $86 \%$ & & & & \\
\hline $55 \% \mathrm{Cl}$ SCCP & $\begin{array}{l}10 \\
.00\end{array}$ & $10.00( \pm 0.19)$ & $100 \%$ & $\begin{array}{l}55 \% \mathrm{Cl} \mathrm{SCCP} \text { and } \\
57 \% \mathrm{Cl} \mathrm{MCCP} \\
(1: 1, \mathrm{v} / \mathrm{v} \\
20 \mathrm{ng} / \mu \mathrm{L})\end{array}$ & 10.00 & $12.43( \pm 4.6)$ & $124 \%$ \\
\hline $63 \% \mathrm{Cl}$ SCCP & $\begin{array}{l}10 \\
.00\end{array}$ & $12.05( \pm 0.14)$ & $120 \%$ & & & & \\
\hline $52 \% \mathrm{Cl} \mathrm{MCCP}$ & $\begin{array}{l}10 \\
.00\end{array}$ & $12.13( \pm 0.89)$ & $121 \%$ & & & & \\
\hline $57 \% \mathrm{Cl} \mathrm{MCCP}$ & $\begin{array}{l}10 \\
.00\end{array}$ & $11.36( \pm 0.71)$ & $114 \%$ & $\begin{array}{l}55 \% \mathrm{Cl} \text { SCCP and } \\
57 \% \mathrm{Cl} \mathrm{MCCP} \\
(1: 1, \mathrm{v} / \mathrm{v} \\
20 \mathrm{ng} / \mu \mathrm{L})\end{array}$ & 10.00 & $12.89( \pm 0.27)$ & $129 \%$ \\
\hline
\end{tabular}

a Accuracy is defined as the percentage ratio of the calculated concentration of CPs and the reference concentration of CPs.

$\mathrm{C}_{12} \mathrm{Cl}_{8}$ isomer, the relative abundance of the $[\mathrm{M}-\mathrm{Cl}]^{-}$ion was $96 \%$. Compared to the sharp drops in $[\mathrm{M}-\mathrm{Cl}]^{-}$abundance at source temperatures of $180^{\circ} \mathrm{C}$ and $200^{\circ} \mathrm{C}$, this drop was negligible at $150^{\circ} \mathrm{C}$. Considering that a low ion source temperature appeared unstable and resulted in contamination, $150^{\circ} \mathrm{C}$ was selected for the remainder of the temperatures.

The fragmentation patterns of the $\mathrm{CP}$ mixtures were found to contain a large number of ions (Table S3, Figs. 2, S6, and S7). Fig. S7 shows the possible fragmentation patterns of two single isomers, namely 1,1,1,3,9,11,11,11-octachloroundecane and $1,1,1,3,10,12,12,12$-octachlorododecane. The results suggest that ions such as $[\mathrm{M}+\mathrm{Cl}]^{-}$generated from $\mathrm{C}_{11} \mathrm{Cl}_{6}$, and $[\mathrm{M}-2 \mathrm{Cl}]^{-}$generated from $\mathrm{C}_{11} \mathrm{Cl}_{9}$, will interfere with the quantitative ion of $\mathrm{C}_{11} \mathrm{Cl}_{8}$. However, as the TOF scan offers information in the full $\mathrm{m} / \mathrm{z}$ range of 50-600, this enables us to select suitable ions for quantification and qualification to distinguish different $\mathrm{CP}$ congener groups.

\subsection{Influence of resolution in $\mathrm{CP}$ analysis}

The relationships between resolution, mass accuracy, and deviation of mass (DM) are elucidated in Eqs. (2) and (3)

Resolution $=\frac{\mathrm{M}}{\mathrm{DM}}$

MassAccuracy $=\frac{M}{\mathrm{DM}} \times 10^{6}$

where $\mathrm{M}$ is the mass-to-charge ratio of the fragment ions, DM is the mass distance between two adjacent peaks, and the mass accuracy is presented in ppm.

As shown in Eqs. (2) and (3), the resolution of a signal is related to the mass of the species. In this case, the majority of CP target ions were in the $\mathrm{m} / \mathrm{z}$ range of $300-500$ (Figs. $S 1, S 2$ ), where TOF resolutions of $10,000-15,000$ could theoretically yield mass accuracies of $5-10 \mathrm{ppm}$. For the 96 quantitation and qualification fragments, a minimum resolution of 3000 was required for the separation of the two closest $\mathrm{m} / \mathrm{z}$ values for the $\mathrm{C}_{12} \mathrm{H}_{16}{ }^{35} \mathrm{Cl}_{7}{ }^{37} \mathrm{Cl}_{2}$ (478.839 Da) and $\mathrm{C}_{17} \mathrm{H}_{28}{ }^{35} \mathrm{Cl}_{6}{ }^{37} \mathrm{Cl}$ (478.9987 Da) fragments (Table 2). However, if considering the different fragmentation patterns and isotopic composition (Table S3), a minimum resolution of $26,000\left(\mathrm{C}_{11} \mathrm{H}_{16}{ }^{35} \mathrm{Cl}_{9}=462.8454 \mathrm{Da}\right.$, and $\mathrm{C}_{11} \mathrm{H}_{14}{ }^{35} \mathrm{Cl}_{8}{ }^{37} \mathrm{Cl}=462.8628 \mathrm{Da}$ ) was required to resolve all $224 \mathrm{~m} / \mathrm{z}$ values with an abundance of above $5 \%$. As previously mentioned, we selected an ion source temperature of $150^{\circ} \mathrm{C}$ to minimize fragmentation patterns other than $[\mathrm{M}-\mathrm{Cl}]^{-}[5,18]$. Indeed, if $[\mathrm{M}-\mathrm{Cl}]^{-}$ could be considered the main fragmentation pattern, then the res- olution requirement will be $8000\left(\mathrm{C}_{12} \mathrm{H}_{16}{ }^{35} \mathrm{Cl}_{7}{ }^{37} \mathrm{Cl}_{2}=478.839 \mathrm{Da}\right.$ and $\mathrm{C}_{17} \mathrm{H}_{28}{ }^{35} \mathrm{Cl}_{6}{ }^{37} \mathrm{Cl}=478.9987 \mathrm{Da}$ ). Thus, the qTOF-HRMS method applied in this study with a resolution of $12,000-15,000$ was suitable for resolving all congener groups of SCCPs and MCCPs.

The quantification performances at 10, 20, 50, 100, 200, and $500 \mathrm{ppm}$ were then compared as shown in Fig. 3. At mass tolerance windows of 10 and $20 \mathrm{ppm}$, the RSD values of some formula groups between different injections were relatively large ( $>10 \%)$, even for the reference standards. At a mass tolerance window of $500 \mathrm{ppm}$, interference was observed by EIC for samples from different environmental matrices (Fig. 4). At mass tolerance windows of 20 and 50 ppm, we got almost the same results. Furthermore, mass tolerance windows of $100 \mathrm{ppm}$ and $200 \mathrm{ppm}$ were also acceptable with weaker matrix effects.

Furthermore, matrix interference was found to exist even following thorough sample pretreatment procedures [23]. Under the ECNI-LRMS system, SIM combined the retention time window to eliminate self-interference. However, this approach did not yield satisfactory results (see Fig. 4: EIC at $\pm 0.5 \mathrm{amu}$ ) as baseline separation of the components could not be achieved. As shown in Fig. 4, interference from the matrix along with $\mathrm{CP}$ self-interference (e.g. $\mathrm{m} / \mathrm{z} 451$ generated by $\mathrm{C}_{10} \mathrm{Cl}_{10}$ and $\mathrm{C}_{15} \mathrm{Cl}_{8}$ ) could be avoided to a large extent with a mass tolerance of $50 \mathrm{ppm}$ upon extracting the accurate mass. In Fig. 4, MCCPs were treated as interference while SCCPs were regarded as the targets. The reverse case (SCCP interference and MCCPs as targets) is displayed in Fig. S9.

Additionally, we tested the deviations of the detected values from the true values at a range of concentrations $(0.25,0.5,1.0,2.5$, $5.0,10,20$, and $50 \mathrm{ng} / \mu \mathrm{L}$ ) using binary mixture standards of $55.5 \%$ $\mathrm{Cl} \mathrm{SCCP}$ and $57 \% \mathrm{Cl}$ MCCP. We found that self-interference between SCCP and MCCP was acceptable (Fig. S4).

\subsection{Response factor and chlorination}

The quantitative method based on the linearity of the response factor and chlorination compensated for the difference in response factors between the reference $\mathrm{CP}$ mixtures and the real samples [12], giving satisfactory quantification results even at a high degree of chlorination (e.g., 57\% Cl MCCP; Fig. 1(c)). However, chlorination could not be neglected due to marked changes in the response factor with chlorination (Table 3). However, during MCCP quantification, the calculated sample chlorination exceeded the calculated chlorination range of the MCCP standards. Indeed, the results obtained were either $<0$ or exceedingly high, and so the MCCPs could not be precisely quantified in any of these samples. The con- 
Table 2

Accurate masses of MCCP and SCCP formulation groups which generated fragmentation ions with the same nominal mass and the D-value between the two ions.

\begin{tabular}{|c|c|c|c|c|c|}
\hline Nominal mass & Formula group & Accurate mass & Formula group & Accurate mass & D-value $\left(p^{2}{ }^{a}\right)$ \\
\hline 417 & $\mathrm{C}_{10} \mathrm{Cl}_{9}$ & 416.8472 & $\mathrm{C}_{15} \mathrm{Cl}_{7}$ & 417.0063 & 382 \\
\hline 451 & $\mathrm{C}_{10} \mathrm{Cl}_{10}$ & 450.8082 & $\mathrm{C}_{15} \mathrm{Cl}_{8}$ & 450.9674 & 353 \\
\hline 431 & $\mathrm{C}_{11} \mathrm{Cl}_{9}$ & 430.8628 & $\mathrm{C}_{16} \mathrm{Cl}_{7}$ & 431.0220 & 369 \\
\hline 465 & $\mathrm{C}_{11} \mathrm{Cl}_{10}$ & 464.8239 & $\mathrm{C}_{16} \mathrm{Cl}_{8}$ & 464.9830 & 342 \\
\hline 445 & $\mathrm{C}_{12} \mathrm{Cl}_{9}$ & 444.8785 & $\mathrm{C}_{17} \mathrm{Cl}_{7}$ & 445.0376 & 358 \\
\hline 479 & $\mathrm{C}_{12} \mathrm{Cl}_{10}$ & 478.8395 & $\mathrm{C}_{17} \mathrm{Cl}_{8}$ & 478.9987 & 333 \\
\hline
\end{tabular}

a Part per million.
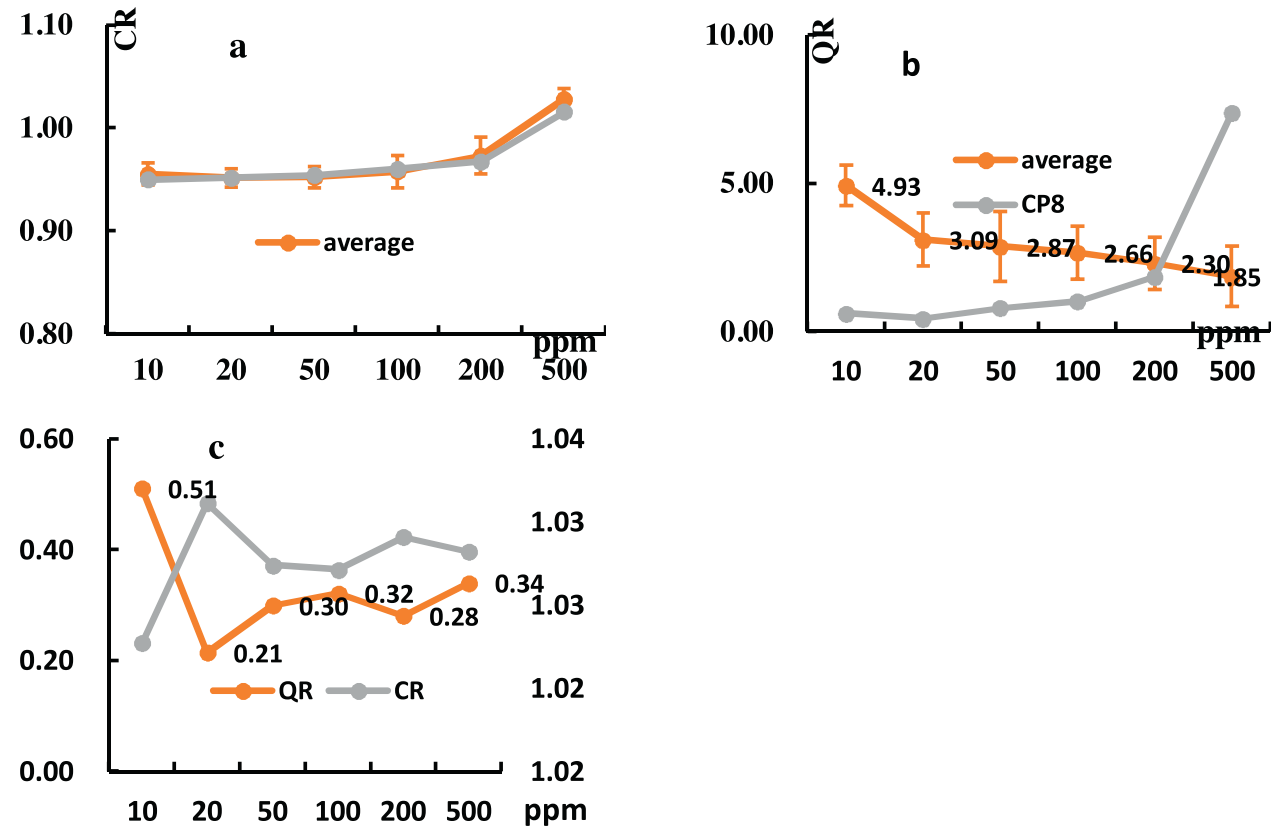

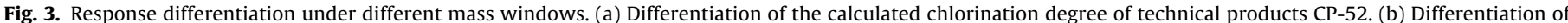
the quantification results of technical products. (c) Differentiation of calculated chlorination degree and quantification results of XAD-based air samples.

Table 3

Calculated chlorination degree (average of three measurements \pm standard deviation) for the different technical CP formulations.

\begin{tabular}{|c|c|c|c|c|}
\hline & Calculated chlorination $^{a}$ & Response factor ${ }^{a}$ & Calculated chlorination ${ }^{\mathrm{b}}$ & Response factor ${ }^{b}$ \\
\hline SCCP $51 \% \mathrm{Cl}$ & $59.5 \% \mathrm{Cl}( \pm 0.12)$ & 0.0084 & $58.8( \pm 0.09)$ & 0.009 \\
\hline SCCP $55 \% \mathrm{Cl}$ & $61.3 \% \mathrm{Cl}( \pm 0.28)$ & 0.031 & $60.5( \pm 0.11)$ & 0.025 \\
\hline SCCP $63 \% \mathrm{Cl}$ & $64.7 \% \mathrm{Cl}( \pm 0.39)$ & 0.16 & $64.0( \pm 0.41)$ & 0.089 \\
\hline $\mathrm{MCCP} 42 \% \mathrm{Cl}$ & $54.0 \% \mathrm{Cl}( \pm 0.31)$ & 0.0029 & & \\
\hline MCCP $52 \% \mathrm{Cl}$ & $57.5 \% \mathrm{Cl}( \pm 0.39)$ & 0.038 & & \\
\hline MCCP $57 \% \mathrm{Cl}$ & $59.6 \% \mathrm{Cl}( \pm 0.27)$ & 0.075 & & \\
\hline
\end{tabular}

a Stands for high resolution TOF mass results.

b Stands for low resolution mass result.

gener profiles of the MCCPs in the four matrices are shown in Fig. S5. In addition, as shown in Fig. 3c, the chlorination degree and the quantification results exhibited opposite trends as the mass accuracy changed, as indicated in Eq. (4):

Content $=\frac{\mathrm{TR}}{\mathrm{RRF}}$

where TR represents the total response in the sample, and RRF is the relative response factor. Furthermore, as illustrated in Fig. S4, the RRF showed a positive linear relationship with chlorination, while the RRF and the determined content (quantification results) varied inversely (Eq. (4)). Thus, the available CP standards were not suitable to account for the chlorination range for samples from the various environmental matrices. Furthermore, the validity of the determined results relied on the inertness (transformation and evolution were insignificant) of the CPs in the environmental samples.

\subsection{Time efficiency and suitability for routine analysis}

Unlike the earlier LRMS method built in our lab [18,19] that requires four separate runs to acquire all necessary SIM ions for identification and quantification, the new HRMS approach requires only one injection. Furthermore, the higher selectivity afforded by HRMS approach allowed effective use of automatic peak integration without significant interference instead of the very time-consuming manual integration required for LRMS data. This combination reduced turnaround time on samples from a few months to a few days.

\subsection{Analysis of environmental samples and comparison between two methods}

Comparison of results from the current HRMS method with the LRMS method previously reported by our group $[18,19]$ is essen- 

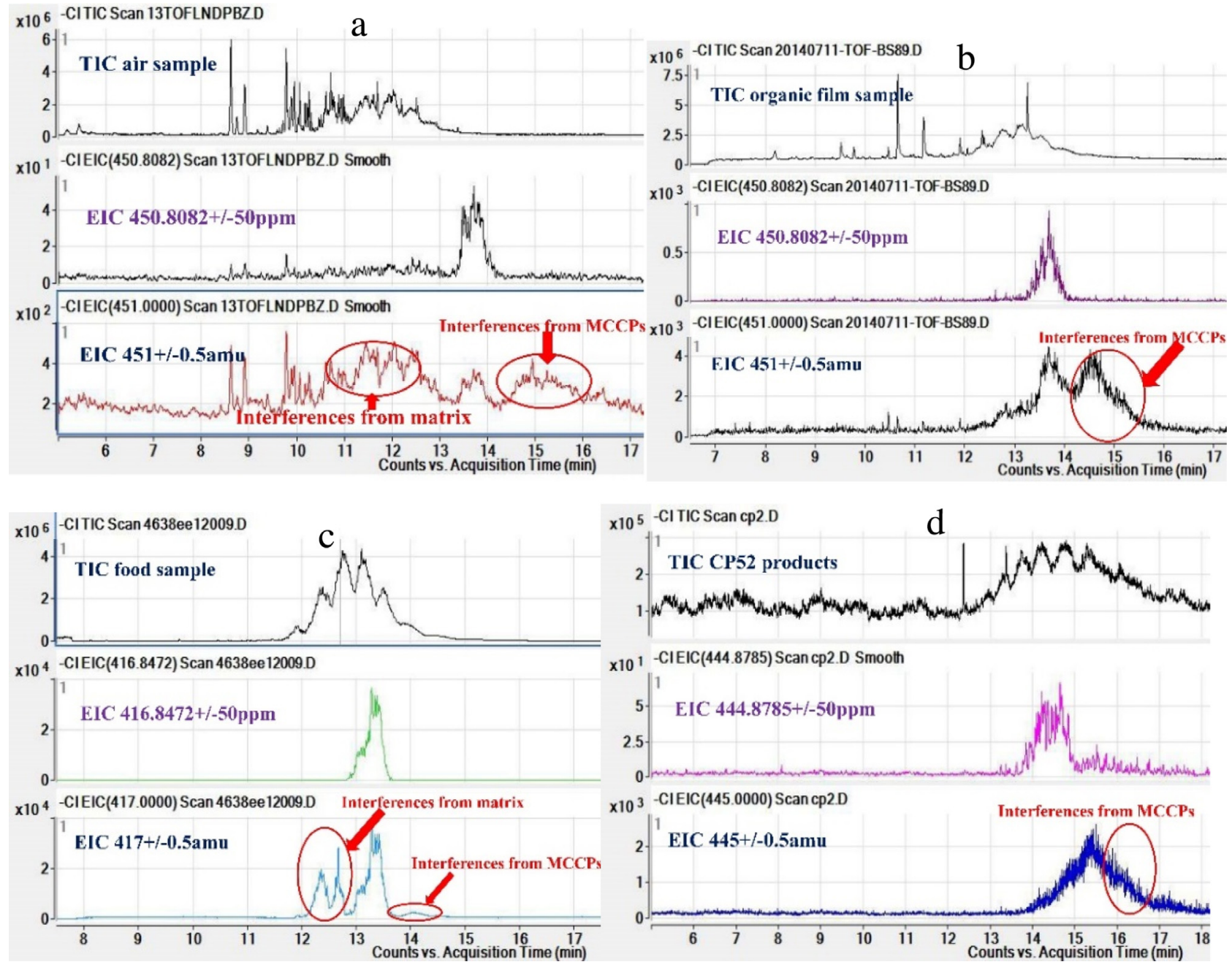

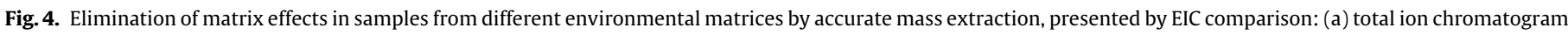

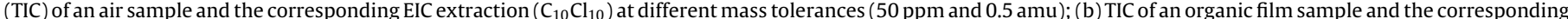

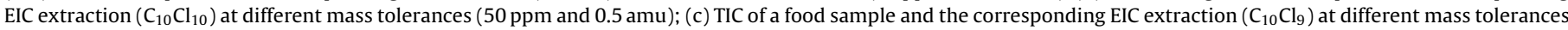
(50 ppm and $0.5 \mathrm{amu})$; (d) TIC of the CP-52 product and the corresponding EIC extraction $\left(\mathrm{C}_{12} \mathrm{Cl}_{9}\right)$ at different mass tolerances (50 ppm and 0.5 amu).

tial to assess improvements in the quality of the $\mathrm{CP}$ environmental measurements [22]. The NCI-qTOF-HRMS method was therefore evaluated to quantify SCCPs and MCCPs in industrial products and in several environmental matrices (food, organic film, and XADbased air samples).

For the food samples, the SCCP concentration ranged from 70 to $73172 \mathrm{ng} / \mathrm{g} \mathrm{dw}$, while for the organic film sample, the SCCP concentration ranged from 97 to $4104 \mathrm{ng} / \mathrm{m}^{2}$. In addition, the SCCP concentration in the XAD-based air samples ranged from $0.04-29 \mathrm{ng} / \mathrm{m}^{3}$, and finally, for the technical products, the SCCP content ranged from 54 to $1651 \mathrm{ng}$ in the $\mathrm{CP}-52$ products at a concentration of $10 \mathrm{ppm}$.

We also compared the SCCP contents and chlorination values obtained using the two different MS methods (Fig. S10). In the organic film samples, similar concentrations were obtained with both methods. More specifically, the SCCP concentrations determined using the novel NCI-qTOF-HRMS method differed from those determined with our GC/ECNI-LRMS method by factors of 0.5-2.2.

In the XAD-based air samples, the concentrations obtained using the NCI-qTOF-HRMS method were generally lower than those obtained using the GC/ECNI-LRMS method, with the exception of two cases (i.e., an extremely low content $(13 \mathrm{bz})$ and an extremely high content (14 dppl)). The SCCP concentrations determined by
NCI-qTOF-HRMS differed from those obtained by GC/ECNI-LRMS by factors of $0.19-0.92$.

In contrast, the results obtained for the food samples varied due to differences in matrix effects. The SCCP concentrations determined by NCI-qTOF-HRMS differed from those obtained by GC/ECNI-LRMS by factors of 0.16-2.55.

Furthermore, for the industrial $\mathrm{CP}$ products, the concentrations obtained using the NCI-qTOF-HRMS method were generally higher than those obtained using the GC/ECNI-LRMS method, with the exception of CP8 (CP52 products provided by Hangzhou Electrochemical. See Supplementary material Sample Collection and Preparation section), which had a very low SCCP content. The SCCP concentrations measured by NCI-qTOF-HRMS differed from those measured by GC/ECNI-LRMS by factors of 3.79-6.05.

To further investigate the reasons for the differences in results obtained using the two methods, individual formula group contents of the SCCPs obtained using both the NCI-qTOF-HRMS method and the ECNI-LRMS method were compared (see Fig. 5). This comparison showed that in air, SCCPs containing fewer chlorine atoms and shorter chain lengths were predominant, whereas the reverse was true for the technical products. As mentioned above, at high resolutions, the obtained content of CPs containing fewer chlorine atoms was lower. Therefore, for the lighter components found in XADbased air samples, the content determined by NCI-qTOF-HRMS was 


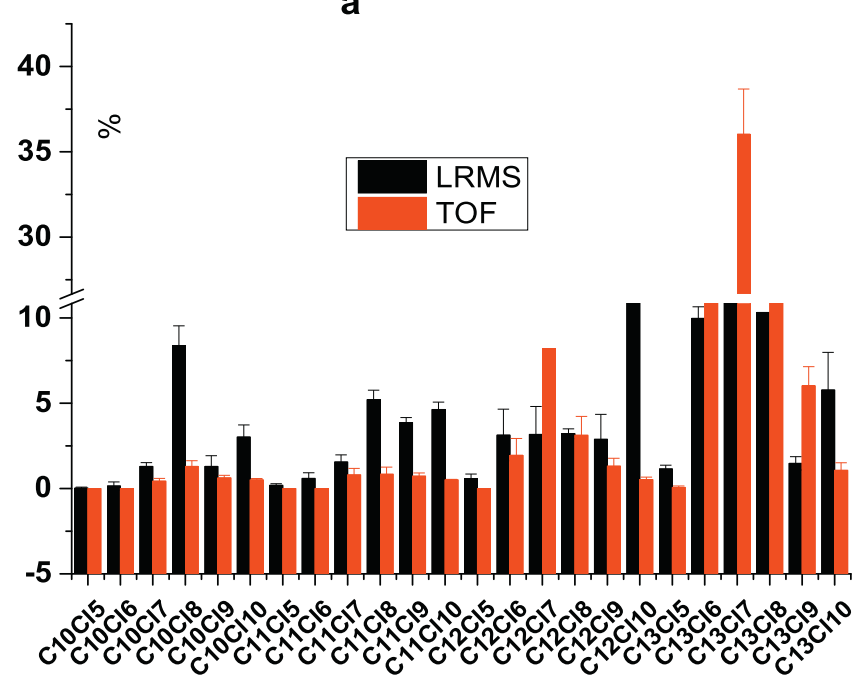

b

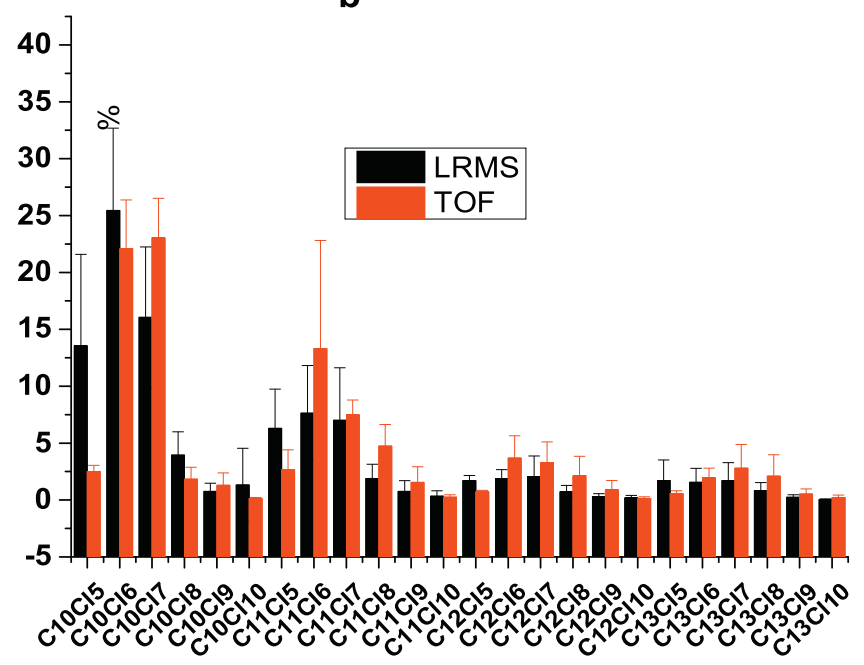

Fig. 5. Comparison of SCCP individual congener contents obtained using the NCITOF-HRMS method (gray bars) and the ECNI-LRMS method (black bars) for (a) congener group distribution of SCCPs in industrial products, and (b) congener group distribution of SCCPs in XAD-based air samples.

higher, while for the heavier components found in technical products, the content determined by NCI-qTOF-HRMS was lower (Fig. 5). However, the differences in the absolute amounts obtained did not represent significant deviations from the true values. As discussed above, CPs with varying chlorine contents exhibited varying response patterns under different instrumental conditions, and this deviation was mainly a result of the varying degrees of chlorination.

The calculated chlorine contents obtained using the $\mathrm{NCI}-$ qTOF-HRMS method were generally higher than the degrees of chlorination calculated using the GC/ECNI-LRMS method, with the exception of industrial CP products and two food samples (potato 2 and razor 1). For SCCPs, in XAD-based air samples, the differences in the chlorination degrees obtained with the two independent methods ranged from $1.3 \%$ to $4.2 \% \mathrm{Cl}$, while for the technical products, these differences ranged from $0.0 \%$ to $4.4 \% \mathrm{Cl}$. This variation could be accounted for by the low content of CPs bearing fewer chlorine atoms (CPs that generate ions with a mass charge ratio of $\sim 300$ ), as determined under a high resolution (in the SIM of the LRMS, interference occurred at $m / z \sim 300$ ).
In this study, analyses of MCCPs were conducted using only the NCI-qTOF-HRMS system. Furthermore, due to a shortage of available data in the literature, no inter-lab comparison results are currently available for MCCPs. Results from our study showed that the MCCP concentration in air was relatively low compared to other matrices, with a content ranging from 0.04 to $0.89 \mathrm{ng} / \mathrm{m}^{3}$ being obtained for the XAD-based samples (the passive air samplers were placed in the Sejila Mountains in Tibet Plateau for $1 \mathrm{yr}$ ). MCCP contents in the organic film samples ranged from $1.37 \mu \mathrm{g} / \mathrm{m}^{2}$ to $18.2 \mu \mathrm{g} / \mathrm{m}^{2}$, while in the food samples, the MCCP levels were between $603 \mathrm{ng} \mathrm{g}^{-1}$ and $7478 \mathrm{ng} \mathrm{g}^{-1}$.

For the industrial products, the concentrations of $\sum \mathrm{CPs}$ (SCCPs + MCCPs) were in the range of 3796-6235 ng in 6CP-52 products (CP2, СP3, СP4, СP5, СР7, CP8; detailed information of these products was provided in Supplementary material) of $10 \mathrm{ng} / \mu \mathrm{L}$ (in which the total amount of $\sum$ CPs should be $2000 \mathrm{ng}$ ), which indicated that the results are overestimated. The possible reason for that is that all the calculated chlorination degrees of the industrial products were at the low end of the calibration curve of chlorine content vs response of MS. As we explained earlier in Eq. (4), the chlorination degree was inversely correlated with the quantification results, the lower chlorination degrees in industrial products than that in environmental matrices might result in the overestimation of $\mathrm{CP}$ concentrations. The results implied that more specific reference standards with wider chlorination range should be synthetized to bulid more accurate quantified and qualified $\mathrm{CP}$ methods for different matrices.

\section{Conclusions}

The reported GC-QTOF-MS method offers a number of benefits over established GC/ECNI-LRMS methods for the analysis of CPS in environmental samples. This novel method was especially efficient in the simultaneous analysis of SCCPs and MCCPs in complex environmental samples, and was efficient in eliminating CP selfinterference by accurate mass extraction. The results obtained for different environmental samples showed that the high-resolution TOF-MS method was capable of reducing interferences from different matrices. In addition, the GC-QTOF-MS method showed a comparable linear dynamic range and detection limits with previous method, along with an improved accuracy. Moreover, this method was suitable for the analyses of large sets of samples due to its efficiency in both the analysis time and quantification processes. Further application of this GC-QTOF-MS method should be examined to achieve more accurate analysis of CPs in different matrices.

\section{Acknowledgments}

We thank the National Basic Research Program of China (2015CB453102), the National Natural Science Foundation of China $(21477141,21321004,21337002)$, and the Strategic Priority Research Program of the Chinese Academy of Science (XDB14010400, YSW2013A01) for financial support. We also acknowledge Ms. Wenwen Wang for her support with MS.

\section{Appendix A. Supplementary data}

Supplementary data associated with this article can be found, in the online version, at http://dx.doi.org/10.1016/j.chroma.2016.04. 081.

\section{References}

[1] The Handbook of Environmental Chemistry, in: D.C.G. Muir, G.A. Stern, G.T. Tomy, J. Paasivirta (Eds.), Springer, New York, 2000. 
[2] European Commission, Directive 2000/60/EC of the European Parliament and of the Council of 232000 establishing a framework for Community action in the field of water policy, Official J. Eur. Commun. (2000).

[3] J. De Boer, The Handbook of Environmental Chemistry, Springer, Berlin Heidelberg, 2016, 2010.

[4] L.M. Van Mourik, P.E.G. Leonards, C. Gaus, J. De Boer, Recent developments in capabilities for analysing chlorinated paraffins in environmental matrices: a review, Chemosphere 136 (2015) 259-272.

[5] G.T. Tomy, G.A. Stern, D.C.G. Muir, A.T. Fisk, C.D. Cymbalisty, J.B. Westmore, Quantifying $C_{10}-C_{13}$ polychloroalkanes in environmental samples by high-resolution gas chromatography/electron capture negative ion high-resolution mass spectrometry, Anal. Chem. 69 (1997) 2762-2771.

[6] G.T. Tomy, G.A. Stern, Analysis of $\mathrm{C}_{14}-\mathrm{C}_{17}$ polychloro-n-alkanes in environmental matrixes by accelerated solvent extraction-high-resolution gas chromatography/electron capture negative ion high-resolution mass spectrometry, Anal. Chem. 71 (1999) 4860-4865.

[7] M. Reth, M. Oehme, Limitations of low resolution mass spectrometry in the electron capture negative ionization mode for the analysis of short- and medium-chain chlorinated paraffins, Anal. Bioanal. Chem. 378 (2004) $1741-1747$.

[8] P. Korytar, J. Parera, P.E.G. Leonards, F.J. Santos, J. De Boer, U.A. Brinkman, Characterization of polychlorinated $n$-alkanes using comprehensive two-dimensional gas chromatography-electron-capture negative ionization time-of-flight mass spectrometry, J. Chromatogr. A 1086 (2005) 71-82.

[9] D. Xia, L.R. Gao, S. Zhu, M.H. Zheng, Separation and screening of short-chain chlorinated paraffins in environmental samples using comprehensive two-dimensional gas chromatography with micro electron capture detection, Anal. Bioanal. Chem. 406 (2014) 7561-7570.

[10] M.L. Nilsson, S. Bengtsson, H. Kylin, Identification and determination of chlorinated paraffins using multivariate evaluation of gas chromatographic data, Environ. Pollut. 163 (2012) 142-148.

[11] S. Geiß, J.W. Einax, S.P. Scott, Determination of the sum of short chain polychlorinated n-alkanes with a chlorine content of between 49 and $67 \%$ in water by GC-ECNI-MS and quantification by multiple linear regression, Clean: Soil Air Water 38 (2010) 57-76.

[12] M. Reth, Z. Zencak, M. Oehme, New quantification procedure for the analysis of chlorinated paraffins using electron capture negative ionization mass spectrometry, J. Chromatogr. A 1081 (2005) 225-231.
[13] M. Coelhan, Determination of short-chain polychlorinated paraffins in fish samples by short-column GC/ECNI-MS, Anal. Chem. 71 (1999) 4498-4505.

[14] O. Koh, W. Rotard, W.H.P. Thiemann, Analysis of chlorinated paraffins in cutting fluids and sealing materials by carbon skeleton reaction gas chromatography, Chemosphere 47 (2002) 219-227.

[15] S.M. Steinberg, D.W. Emerson, On-line dechlorination-hydrogenation of chlorinated paraffin mixtures using GC and GC/MS, Environ. Monit. Assess. 184 (2012) 2119-2131.

[16] Z. Zencak, M. Oehme, Chloride-enhanced atmospheric pressure chemical ionization mass spectrometry of polychlorinated $n$-alkanes, Rapid Commun. Mass Spectrom. 18 (2004) 2235-2240.

[17] C. Bogdal, T. Alsberg, P.S. Diefenbacher, M. Macleod, U. Berger, Fast quantification of chlorinated paraffins in environmental samples by direct injection high-resolution mass spectrometry with pattern deconvolution, Anal. Chem. 87 (2015) 2852-2860.

[18] L.X. Zeng, T. Wang, W.Y. Han, B. Yuan, Q.A. Liu, Y.W. Wang, G.B. Jiang, Spatial and vertical distribution of short chain chlorinated paraffins in soils from wastewater irrigated farmlands, Environ. Sci. Technol. 45 (2011) 2100-2106.

[19] T. Wang, S.L. Han, B. Yuan, L.X. Zeng, Y.M. Li, Y.W. Wang, G.B. Jiang, Summer-winter concentrations and gas-particle partitioning of short chain chlorinated paraffins in the atmosphere of an urban setting, Environ. Pollut. 171 (2012) 38-45.

[20] S. Ong, R.A. Hites, Why are electron capture negative ion mass spectra not reproducible? An ion source problem, J. Am. Soc. Mass Spectrom. 4 (1993) 270-277.

[21] G.T. Tomy, S.A. Tittlemier, G.A. Stern, D.C.G. Muir, J.B. Westmore, Effects of temperature and sample amount on the electron capture negative ion mass spectra of polychloro-n-alkanes, Chemosphere 37 (1998) 1395-1410.

[22] E. Sverko, G.T. Tomy, C.H. Märvin, D.C.G. Muir, Improving the quality of environmental measurements on short chain chlorinated paraffins to support global regulatory efforts, Environ. Sci. Technol. 46 (2012) 4697-4698.

[23] L.G. Chen, Y.M. Huang S. Han, Y.B. Feng, G. Jiang, C.M. Tang, Z.X. Y. W. Zhan, M. Liu, S.K. Zhang, Sample pretreatment optimization for the analysis of short chain chlorinated paraffins in soil with gas chromatography-electron capture negative ion-mass spectrometry, J. Chromatogr. A 1274 (2013) 36-43. 